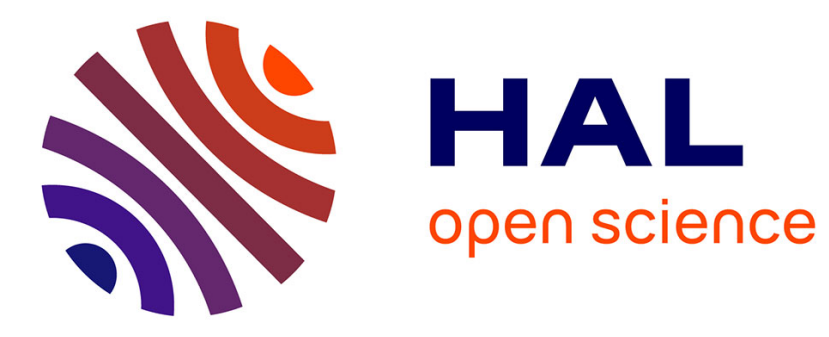

\title{
L'extension de la traçabilité dans le secteur agro-alimentaire
}

Céline Granjou, Egizio Valceschini

\section{To cite this version:}

Céline Granjou, Egizio Valceschini. L'extension de la traçabilité dans le secteur agro-alimentaire. Terrains et Travaux : Revue de Sciences Sociales, 2005, 9, pp.73-89. halshs-00382816

\section{HAL Id: halshs-00382816 https://shs.hal.science/halshs-00382816}

Submitted on 14 May 2009

HAL is a multi-disciplinary open access archive for the deposit and dissemination of scientific research documents, whether they are published or not. The documents may come from teaching and research institutions in France or abroad, or from public or private research centers.
L'archive ouverte pluridisciplinaire HAL, est destinée au dépôt et à la diffusion de documents scientifiques de niveau recherche, publiés ou non, émanant des établissements d'enseignement et de recherche français ou étrangers, des laboratoires publics ou privés. 
Granjou Céline

Valceschini Egizio

L'extension de la traçabilité dans le secteur

agro-alimentaire

Une nouvelle norme de régulation de la production

\section{Introduction}

Questionnement

Avec l'affaire de la vache folle et la controverse sur les OGM émerge un discours récurrent sur la mise en place de la traçabilité dans le secteur agro-alimentaire, dont la caractéristique est d'être porté tant par les associations de défense des consommateurs que par l'Etat et les producteurs eux-mêmes. On assiste ainsi non seulement à une extension des procédures de traçabilité, mais également à un réinvestissement de sens dans cette notion, qui tend à devenir, d'une pratique de suivi et de gestion interne aux entreprises (Cochoy, Garel et de Terssac, 1998 ; Rot, 1998), un outil à grande échelle d'information et de contrôle (Torny, 1998 ; Cochoy, 2002a). Refusant de réduire la traçabilité à une rhétorique publicitaire, cet article analyse, à partir d'une approche socio-économique, son extension dans le secteur agro-alimentaire : sur quels supports matériels et quelles sources d'autorité se fonde l'organisation systématique des activités productives autour de la traçabilité? Quel est le sens de la traçabilité pour les acteurs qui la mettent en oeuvre ? Quelle(s) exigence(s) normative(s) ressortent de la généralisation de ces investissements dans la traçabilité ?

Pour répondre à ces questions, nous avons fait le choix de nous interroger sur le contenu et le sens des investissements que suppose la traçabilité chez les acteurs de la production. Nous entendons par " acteurs de la production » à la fois les acteurs industriels au sens large (producteurs agricoles, transformateurs, distributeurs) et les acteurs tiers intervenant dans la régulation des activités de production (services de 1'Etat, laboratoires d'analyses, sociétés de certification). Cette approche, centrée sur les producteurs plutôt que sur les consommateurs, prend acte des nombreux travaux qui mettent en doute la pertinence, du point de vue des consommateurs, des nouveaux étiquetages permis par la traçabilitéi. Si la traçabilité permet certes de gérer de nouveaux critères de qualification des produits, nous considérons, suivant en cela Franck Cochoy (2002b), que ces critères reposent sur des modifications des réseaux sociotechniques de production : ce sont ces modifications en amont qu'il appartient au chercheur de mettre à jour s'il veut éclairer les dispositifs non-marchands qui sous-tendent et conditionnent les échanges économiques.

Le choix de l'observation parallèle des filières concernées par la crise de la vache folle et par la controverse sur les OGM se justifie alors par la possibilité de mettre à jour les raisons poussant les producteurs à la systématisation d'une organisation reposant sur la traçabilité, dans des contextes qui, s'ils contribuent à une « crise de confiance » générale dans le secteur alimentaire, restent pourtant fort différents. Dans le cas de la viande bovine, la mise en place de la traçabilité doit permettre de conserver certaines caractéristiques de l'animal d'origines tout au long des étapes d'élevage, abattage, découpe et transformation, tandis que, dans le cas des OGM, la traçabilité vise moins à transmettre une information déjà disponible qu'à apporter des preuves de l'absence d'OGM (afin d'être en situation légale de ne pas étiqueter le produit comme contenant des OGM) : il s'agit alors à la fois de garantir le caractère non génétiquement modifié des semences d'origine et de garantir l'absence de contamination lors des diverses opérations de 
transformation. Cette mise en parallèle nous permettra de mettre en question l'identité même de la notion de traçabilité, en pointant différents modèles de mise en oeuvre d'une nouvelle norme orientant les activités de production agro-alimentaire.

Démarche d'enquête

Nous nous sommes fondés sur une enquête visant à analyser la réorganisation des filières viande bovine, maïs et sojat. Nous avons considéré que cette réorganisation renvoyait non seulement à un système de règles formelles réorientant les pratiques, mais également à un nouvel « ordre moral » qui correspond à la manière dont les acteurs donnent sens à ces normes, se les approprient ou éventuellement s'en écartent (Friedberg, 1996). Nous nous sommes donc fondés sur une trentaine d'entretiens semi-directifs effectués avec les acteurs de la production et interprétés selon une lecture compréhensive. L'échantillonnage s'est fondé sur une logique d'exemplarité au détriment d'une logique d'exhaustivité, en privilégiant la diversité des entreprises du point de vue notamment de leur stade dans la filières. Ce travail a permis à la fois de reconstituer la circulation des écrits supports de la traçabilité, de pointer le rôle crucial des acteurs tiers, qui exercent une fonction de contrôle et de sanction, et de comprendre les modes d'engagement des acteurs industriels dans la traçabilité.

Si la traçabilité remplit une fonction de certification grâce à une circulation de documents validée par l'autorité d'acteurs tiers, l'engagement des industriels dans ces démarches de gestion est ambigu : tout en dénonçant leur caractère paperassier et conventionnel, les industriels s'engagent pour beaucoup avec zèle dans ces procédures, indiquant combien la traçabilité remplit en même temps une fonction de défense et valorisation des activités agro-industrielles face au développement des risques médiatiques. Les deux situations étudiées suggèrent cependant des formes différenciées de conception et de mise en oeuvre de cette norme d'anticipation de la contestation des activités agro-alimentaires (Hommel, 2001).

\section{La traçabilité : supports matériels et sources d'autorité}

La traçabilité vise la transmission et la certification de certaines informations tout au long des étapes de fabrication des produits : il ne s'agit pas seulement de transmettre une information sur l'entité d'origine ou sur un processus, il s'agit également de la certifier, de la garantir en s'en portant caution. Nous explicitons dans cette première partie le fonctionnement de ce dispositif de certification, en montrant qu'il repose d'une part sur la circulation d'un ensemble d' " écrits de traçabilité » et d'autre part sur l'intervention de centres d'autorité qui valident et cautionnent le processus.

Une prolifération de supports d'inscription

Dans le cas de la production de viande bovine, le dispositif de traçabilité repose d'abord sur l'identification des animaux et sur l'enregistrement de leurs changements de propriétaire à l'aide d'une sorte de carte d'identité, le Document d'Accompagnement Bovin (qui correspond aux boucles portées par les animaux). Les enregistrements sont assurés à la fois par la Banque de Données Nationale d'Identification (BDNI), et par l'Etablissement Départemental de 1'Elevage qui tient un Livre des Bovins. Le dispositif repose ensuite sur la correspondance entre les Documents d'Accompagnement Bovins et les numéros attribués aux carcasses et aux morceaux, qui sont également transmis et enregistrés à la Banque de Données Nationales.

La traçabilité des OGM renvoie quant à elle à l'obligation d'étiquetage de tout produit dont un ingrédient contient plus de $0,9 \%$ 
d'OGM7. Dès la fin des années 90, la volonté de ne pas être contraint à étiqueter les produits a poussé les grands distributeurs et fabricants à une série de stratégiess: élimination de certains ingrédients à base de maïs ou de soja, substitution à l'aide d'autres matières premières, et enfin imposition aux fournisseurs d'une série d'exigences visant à obtenir des garanties concernant le caractère non génétiquement modifié des ingrédients fournis. La traçabilité repose alors sur une série de certificats, contrats et cahiers des charges transmis de fournisseur à client, et conservés par les opérateurs eux-mêmes ainsi que par des organismes privés tiers, les sociétés de certification.

Le fonctionnement de la traçabilité renvoie ainsi aux analyses de Béatrice Fraenkel (1995) concernant les « écrits d'action », fiches techniques et autres bons de livraison utilisés dans les entreprises au quotidien de leur travail. Dans la lignée des analyses de Jack Goody (1986) concernant les liens entre les supports techniques de l'écriture et les modes d'organisation sociale, Béatrice Fraenkel montre que les « écrits d'action », destinés à faciliter la production plutôt qu'à informer, relèvent d'un mode d'écriture où la fonction d'information et les supports classiques d'inscription se dissolvent au profit d'une visée de certification et d'assignation, assurée par un marquage codifié sur des supports mobiles. La chaîne que constituent les écrits de traçabilité tout au long des étapes de la filière viande bovine et des filières maïs et soja témoigne bien de ce mécanisme de certification, à la nuance près que deux types de supports y sont mobilisés : des supports circulant assurant la transmission de l'information d'un opérateur à l'autre (Document Bovin, bons de livraison et factures, certificats de nature des semences, résultats d'analyse), et des supports fixes, conservés par les professionnels, mémorisant l'évolution de l'identité des objets en assurant les correspondances de numérotations (Livre des Bovins, Base de Données Nationale, fiches de découpe, registres professionnels, résultats des plans de contrôle). L'association entre supports mobiles et supports fixes permet l'intervention d'instances tierces qui assurent un rôle crucial de validation, de contrôle et de sanction, donnant valeur à grande échelle à la fonction de certification - à la différence des « écrits d'action » de Béatrice Fraenkel qui n'assurent une fonction de certification qu'à l'échelle de l'entreprise où ils restent confinés.

Deux types de sources d'autorité :

réinvestissement étatique versus arrangements privés La traçabilité est ainsi loin d'être seulement une « organisation ponctuelle et circulante, qui voyage bien fixée sur le dos des produits » (Cochoy, 2002) : l'organisation circulante suppose simultanément la mobilisation de sources d'autorité qui donnent à la traçabilité une véritable portée de régulation des activités de production. Nous allons voir que ce rôle de validation est assuré dans le cas de la viande bovine par l'Etat, renforcé dans ses prérogatives régaliennes de contrôle et de sanction, et par des acteurs privés dans le cas des OGM, palliant alors un déficit d'expertise et de capacité de contrôle étatiquess.

La réglementation a formalisé de façon beaucoup plus précoce et précise les obligations des opérateurs de la filière bovine que celles des filières maïs et soja. D'une façon générale, l'investissement de 1'Etat est beaucoup plus affirmé dans la filière bovine - la crise de la vache folle ayant mis très directement en cause la responsabilité institutionnelle de certains ministères- que dans le cas des OGM. En témoigne par exemple le statut particulier des laboratoires d'analyse consacrés à la détection de l'ESB, soumis à une procédure d'agrément public et répertoriés dans une liste officielle, formant 
ainsi le fondement des capacités d'expertise de l'Etat. A contrario, la détection des OGM repose essentiellement sur des laboratoires privés qui sont l'objet d'une accréditation volontaire, à partir d'un système de normes co-produites par les divers acteurs concernés (au sein de l'AFNOR, Association Française de Normalisation et avec l'intervention du Cofrac, Comité Français d'Accréditation). Par ailleurs, la fonction étatique de contrôle et de sanction de l'Etat a été réaffirmée dans le secteur de la viande bovine à travers non seulement de nouveaux plans de contrôle annuels mais également un accroissement du pouvoir de sanction des Services Vétérinaires et des Services de la Répression des Fraudes (Granjou, 2004). Au niveau de l'élevage d'abord, la correction de l'identification des bovins donne lieu à des contrôles accrus et à des sanctions plus lourdes de la part des Services Vétérinaires ; désormais, la perte des deux boucles d'oreille portées par un animal est susceptible de conduire à sa destruction :

"Et puis avant, on ne mettait pas des PV comme ça pour l'identification dans les élevages. Avant, les contrôles dans les élevages n'avaient lieu que par rapport aux primes qui étaient versées; tandis que là, ils [les éleveurs] n'ont rien demandé, sachant qu'il sont quand même contrôlés à l'abattoir, qu'ils risquent de pas pouvoir vendre leurs bêtes, là, même chez eux ils peuvent pas les détenir mal identifiées, même chez eux! 》 (un agent des Services Vétérinaires).

De même, l'abattoir est désormais soumis à une surveillance systématique inédite. La présence d'un vétérinaire-inspecteur est maintenant nécessaire pour superviser les prélèvements de cerveau de chaque animal abattu en vue du test de détection de 1'ESB, tandis que la correction des documents de traçabilité est très strictement surveillée par ces mêmes inspecteurs :

"Toutes les erreurs sont relevées, l'abattoir est un goulet d'étranglement terrible, il n'y a maintenant plus rien qui passe. L'abattoir travaille maintenant sous un contrôle permanent de l'administration, avec l'inspection vétérinaire [...] Donc maintenant à l'abattoir, il n'y a plus rien qui passe : un chiffre de travers, et paf, l'animal est coincé, consigné, regardé dans tous les sens, c'est tout juste s'il n'y a pas une mission de 15 inspecteurs qui viennent la regarder! " (un directeur d'abattoir) Cette surveillance, qui s'ajoute aux exigences récentes de mise aux normes européennes des bâtiments, crée des contraintes techniques et organisationnelles qui mettent en difficulté les petites structures d'abattage (concernant par exemple l'organisation de la présence du vétérinaire sur des durées courtes réparties dans la semaine).

La Banque de Données Nationale d'Identification, instance officielle centralisant des informations auparavant éparpillées dans les départements selon des formes et des codes divers a par ailleurs un rôle crucial de contrôle centralisé, dans la mesure où elle archive les mouvements des animaux au moyen des informations que lui envoient éleveurs, marchands de bestiaux et abattoirs, et repère les incohérences éventuelles de ces échanges.

Si la réorganisation de la filière bovine repose ainsi sur une traçabilité centralisée, légiférée, surveillée et sanctionnée par 1'Etat, la fiabilité de la qualification «non-OGM » des produits échangés dans les filières maïs et soja renvoie en revanche à une fonction de validation et de contrôle assurée par des acteurs privés. La traçabilité s'impose ici à la plupart des acteurs du fait des exigences de preuves de la part des distributeurs et les grands fabricants, qui sont répercutées en cascade d'aval vers l'amont de la filière ${ }_{10}$. Ces exigences ont rapidement conditionné l'accès même au marché, les grands amidonniers s'étant par exemple mis à attribuer des notes 
aux coopératives agricoles en fonction de la façon dont elles remplissent les exigences de traçabilité : dans la mesure où un nombre suffisant de coopératives se voit attribuer la meilleure note, les débouchés disparaissent pour celles qui auraient des pratiques de traçabilité jugées inférieures.

Ces exigences ont ainsi donné lieu à des arrangements locaux et privés entre fournisseurs et clients, reposant sur la constitution de dossiers de traçabilité et incluant souvent le recours à des sociétés de certification. L'intervention de la Répression des Fraudes reste en effet insuffisante à garantir la loyauté des engagements pris entre opérateurs, dans la mesure d'une part où ceux-ci outrepassent les exigences de la réglementation (informer sur la présence d'OGM) pour viser une absence d'OGM, et où les enquêtes menées par les Fraudes paraissent insuffisantes en nombre (elles concernent essentiellement les filières alléguant l'utilisation de semences non génétiquement modifiées telles Céréal) comme en capacités d'expertise (le nombre de tests de détection réalisés dans le cadre des services de la Répression des Fraudes est inférieur au nombre de tests réalisés annuellement par une petite coopérative agricole). Des sources d'autorité privées garantissent le respect des engagements pris entre fournisseurs et clients : si certains grands industriels possèdent une réputation qui leur confère un statut et des ressources suffisantes pour contrôler eux-mêmes leurs fournisseurs voire les fournisseurs de leurs fournisseurs, d'autres font appel à la caution jugée supérieure de sociétés de certification, telles Bureau Veritas ou la Société Générale de Surveillance, dont les activités dans le domaine des OGM se sont développées depuis la fin des années 90 (Granjou et Valceschini, 2004)11.

La description du fonctionnement de la traçabilité dans les deux types de filière montre ainsi des dispositifs différents de par les relations qu'ils supposent entre Etat et professionnels. Si la traçabilité de la viande bovine relève du modèle d'un Etat prescripteur et contrôleur, celle des OGM renvoie bien plus à un modèle de professionnalisme, où les industriels ont une grande autonomie de moyens pour assurer les objectifs globaux fixés par l'Etat. Nous allons voir que cette disparité de fonctionnement s'accompagne d'une appropriation différente des dispositifs par les acteurs qui s'y investissent, même si le référent principal reste celui d'une nouvelle nécessité de défense et valorisation des activités agroalimentaires.

\section{La traçabilité : « modèle du parapluie » ou dispositif de défense et valorisation des activités agro-alimentaires ?}

Le fonctionnement de la traçabilité induit dans tous les cas une activité particulière de la part des acteurs des entreprises, censés tenir à jour les enregistrements, fournir les informations demandées, transmettre les documents nécessaires. Nous décrirons dans un premier temps ce développement de cette gestion administrative, plus ou moins inédite selon les secteurs, avant de détailler le sens que lui donnent les acteurs qui s'y investissent.

Le développement d'une gestion bureaucratique Le développement de la gestion administrative pèse tout particulièrement sur la filière viande bovine, restée traditionnelle et peu touchée par les démarches de différenciation de produits agroalimentaires.

Dans la filière, ce sont l'amont agricole et les petits distributeurs (bouchers) qui voient leurs pratiques particulièrement transformées. Le boucher voit par exemple son métier et les compétences qu'il réclame profondément modifiés par la multiplication des informations codifiées écrites, qui rendent obsolètes l'expérience et le savoir-faire qui permettaient de 
reconnaitre à l'oeil les caractéristiques des bêtes. Aujourd'hui, dans un rayon boucherie de grande surface, le chef boucher aura pour rôle d'assurer l'enregistrement des numéros de lot à la réception des factures, de conserver ces dernières, de vérifier la comptabilité matière (la correspondance des poids du lot reçu et du poids des morceaux emballés provenant de ce lot) ainsi que, s'il est équipé d'un logiciel permettant de suivre en temps réel le découpage et le conditionnement des lots se déroulant dans une salle spéciale, de contrôler depuis l'écran de son ordinateur dans son bureau les données apposées sur les étiquettes. L'abattoir, qui devient le pivot du suivi entre l'animal vivant et les carcasses, doit également s'engager dans des activités bureaucratiques plus importantes, comme le montrent notamment les va-et-vient du « tueur » entre salle d'abattage et bureau du secrétariat, afin de vérifier lui-même la conformité du document édité par le secrétariat avec la numérotation des boucles portées par l'animal, tandis que l'industriel découpant la carcasses assure la tenue de correspondances entre les divers numéros.

Le poids de la gestion administrative apparaît moins inédit dans les filières maïs et soja, composées en partie de très grandes entreprises industrielles habituées aux diverses démarches qualité. Dans le cas des grandes entreprises d'amidonnerie ou de semoulerie, la thématique OGM augmente simplement un peu plus le poids d'une gestion de documents qualité déjà très développée. Les agriculteurs sont quant à eux soumis à une obligation nouvelle de conserver les certificats de semences et de s'engager, par écrit, à ne pas cultiver de semences génétiquement modifiées : les difficultés dont témoigne un responsable de coopérative agricole à recueillir ces documents pour l'ensemble des agriculteurs adhérents suggèrent ici aussi le caractère inédit et contraignant de cette gestion administrative incombant au maillon agricole.

La dénonciation d'un système de preuves conventionnelles Ces nouveaux investissements dans une gestion administrative, qu'elle soit imposée par l'Etat ou par un gros client, sont souvent vécus comme une contrainte vaine, comme une paperasserie inutile par les professionnels $\mathbf{s}_{2}$, qui dénoncent le caractère conventionnel des preuves et des informations transmises.

Les éleveurs rencontrés ou leurs représentants à la Chambre d'Agriculture estiment ainsi que la traçabilité actuelle apporte essentiellement des obligations administratives supplémentaires qui ne se justifient pas par une information pertinente puisque l'animal d'origine reste inconnu : le système de correspondances de numéros ne permet pas de retrouver l'animal dont est issue la viande, mais ouvre seulement la possibilité d'une enquête remontant, à partir des correspondances enregistrées, à un groupe d'animaux dont provient la viande (ce groupe étant d'autant plus vaste que les opérations de mise en lots auront été nombreuses). Un certain nombre d'éleveurs estiment que cette forme de traçabilité ne permet donc pas de valoriser correctement les pratiques d'élevage et se sont regroupés pour s'engager dans des opérations de vente directe à partir de l'achat de structures d'abattage et de découpe : la vente directe court-circuite les maillons industriels et assure sans intermédiaires l'identification de l'animal d'origine ainsi que celle de l'éleveur luimême (Granjou, 2004).

Dans le cas des OGM, les stratégies des grands distributeurs et fabricants visant à ne pas étiqueter leurs produits ont créé une exigence spécifique de preuves quant à la composition des ingrédients, dont les industriels enquêtés ne laissent pas de dénoncer le caractère formel et conventionnel. Ils regrettent tout d'abord que l'existence de méthodes de détection des OGM n'apporte 
pas de garantie définitive, dans la mesure où la normalisation de ces techniques n'est pas encore aboutie et pose donc des problèmes de fiabilité des résultats. De plus, la nouvelle réglementation tend à évincer ce type de preuve technique en exigeant d'étiqueter des produits dans lesquels toute trace d'OGM aurait disparu mais dont les processus de fabrication auraient utilisé des $\mathrm{OGM}_{13}$. Les industriels de l'aval, qui recourent peu aux analyses 14 , soulignent pour leur part l'accumulation de dossiers volumineux de traçabilité (incluant bulletins d'analyse, engagements écrits, certificats et rapports d'audit) dont aucun élément n'a valeur définitive de preuve. Un certain nombre d'acteurs dénoncent ainsi la tendance à toujours «laver plus blanc que blanc »s, entendant par là les exigences croissantes de leurs clients alors que l'absence d'OGM n'est finalement jamais totalement garantie. La dernière réglementation d'étiquetage des OGM est tout particulièrement dénoncée pour son irréalisme en matière de preuve : comment prouver la nonutilisation d'OGM dans les processus si l'on ne peut se fier à la composition finale des produits ? Cela a-t-il même un sens pour le consommateur, qui risque d'être confronté à des produits étiquetés OGM alors qu'ils n'en contiennent pas, et à des produits non étiquetés qui en contiendraient un faible pourcentage ${ }_{16}$ ? Un grand industriel compare ainsi le type de garantie qui pourra être apporté sur les modes de fabrication à celle donnée a posteriori par le rabbin concernant la conformité d'un morceau de viande aux règles religieuses d'abattage. Se plier aux diverses demandes de certificats de la part des clients en fonction de l'actualité des préoccupations sanitaires apparaît d'une façon générale comme une sorte de rituel nécessaire au maintien du marché.

La réappropriation des investissements dans la traçabilité : stratégie du "parapluie » ou nouvelle régulation de la filière Mais de façon paradoxale, les dénonciateurs du caractère bureaucratique et conventionnel des nouvelles procédures font généralement simultanément preuve d'un engagement zélé dans les démarches de traçabilité, quitte pour certains à modifier le sens de la notion. Les éleveurs par exemple, tout en critiquant les contraintes officielles, redéfinissent la notion de traçabilité en fonction de leurs propres intérêts professionnels : la " véritable » traçabilité est assimilée à la proximité entre le producteur et le consommateur, si bien que la plupart des éleveurs revendiquent un engagement ancien pour davantage de traçabilité comprise dans ce sens, et accusent les industriels de chercher à brouiller la visibilité de la qualité du travail effectué en amont afin de ne pas avoir à le rétribuer à sa juste valeur :

"La traçabilité, c'est une relation qu'il y a entre le producteur et le consommateur; tout ce qu'il y a entre, c'est des gens qui, moins il y a de traçabilité, mieux ils se portent. " (un éleveur) Cette compétition entre producteurs agricoles et industriels pour la définition et l'appropriation de la « vraie » traçabilité témoigne ainsi d'une forme de défense des activités de la filière bovine fragilisée par l'affaire de la vache folle, qui repose sur le report des responsabilités des dysfonctionnements potentiels. Tandis que le renforcement des capacités de contrôle de l'Etat sur les pratiques professionnelles tend à prouver le sérieux de l'engagement des pouvoirs publics en renvoyant dans la sphère professionnelle la responsabilité des dysfonctionnements possibles, les acteurs de la filière tendent à s'approprier la traçabilité comme une forme de protection qui défendrait un « monde » professionnel (agricole ou industriel) au détriment d'un autre.

Dans le cas des OGM, les critiques de 1'accumulation de contraintes procédurières sont également loin de justifier une attitude désinvolte 
vis-à-vis de l'investissement dans les procédures de traçabilité : le zèle dont font preuve certaines grandes entreprises pour sélectionner un «bon » laboratoire d'analyse des OGM (procédures de sélection passant au minimum par l'envoi d'un questionnaire et parfois par la réalisation, coûteuse, de tests en aveugle), ou pour constituer de volumineux dossiers de traçabilité, se comprend dans le contexte d'une conscience forte des risques de mise en cause médiatique. Cette conscience est certes davantage le fait d'entreprises d'une certaine taille, engagées dans des démarches actives de préservation de leur réputation. Sans toutefois nécessairement tenir une revue de presse détaillée, les entreprises, quelle que soit leur taille, citent fréquemment la médiatisation des diverses découvertes de produits « contaminés » (maïs Starlinck aux Etats-Unis en septembre 2002, semences génétiquement modifiées en France en été 2002), les publications régulières d'analyses « indépendantes » par les associations de consommateurs 1 , et les actions de Greenpeace. Dans le contexte de cette conscience du risque médiatique lié aux OGM, les professionnels conçoivent ainsi les démarches de traçabilité comme susceptibles d'apporter la preuve, sinon technique (composition des ingrédients), du moins déontologique de leur effort de satisfaire les consommateurs en excluant les OGM de leurs produits. Le recours aux sociétés de certification, que l'on peut rapprocher des dispositifs de confiance décrits par Karpik (1996), est symptomatique de cette attribution d'une signification déontologique à la traçabilité : la certification permet en effet, selon les témoignages des industriels comme des responsables de la certification, bien plus que de garantir les caractéristiques techniques des produits (pourcentage d'OGM contenu), d'apporter une caution des bonnes pratiques de l'industriel (sélection des fournisseurs, choix des laboratoires, réalisation de nettoyages...) et finalement de garantir son engagement actif dans une politique générale d'exclusion des OGM (Granjou et Valceschini, 2004)18. Malgré leur critique de la paperasserie et du caractère formel des preuves apportées, les acteurs se réapproprient ainsi la traçabilité comme un instrument susceptible de garantir une certaine forme de protection de leurs activités face aux mises en cause médiatiques. Cette nouvelle exigence normative se réalise toutefois dans les deux cas étudiés dans des dispositifs qui renvoient à des configurations différentes des relations entre acteurs de la production. La conception de la traçabilité portée par certains éleveurs relève d'une stratégie de revalorisation des activités agricoles au détriment des activités industrielles de la filière, suggérant un modèle de « traçabilité-parapluie » qui ressort également du renforcement de la normalisation et de la surveillance étatiques des activités professionnelles - permettant, en cas de dysfonctionnement, d'attribuer les fautes aux professionnels en dédouanant les pouvoirs publics. Le cas des OGM suggère plutôt, au travers de la généralisation d'arrangements conventionnels et privés passés entre fournisseurs et clients, la systématisation de l'organisation d'une filière en fonction d'une nouvelle norme partagée de "gestion

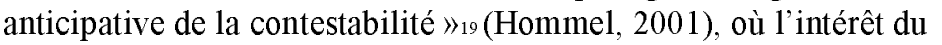
consommateur devient tout autant l'affaire des industriels que celle des pouvoirs publics.

\section{Conclusion}

La mise en place de la traçabilité répond, selon la grande majorité des présentations officielles, à une visée d'information accrue du consommateur dans une logique de transparence. Le parti-pris d'observer la traçabilité du point de vue des producteurs, en mettant en évidence les supports, les pratiques et les significations qui lui sont associés, suggère cependant un autre type de performance, liée 
à la réorganisation des activités de production en fonction de la généralisation d'une norme de « défense et valorisation » des activités agro-industrielles face au risque médiatique. Les deux exemples étudiés montrent que la généralisation de cette norme ne se fait pas selon un modèle d'organisation unique, mais fait appel dans un cas à un renforcement des compétences de contrôle et de sanction de l'Etat, garant de la sécurité sanitaire, et dans l'autre, à la prise en charge volontariste par les acteurs privés d'un parti-pris éthique attribué aux consommateurs. Ces deux modèles d'organisation répondant à une même norme de gestion de la contestabilité des activités agro-alimentaires suggèrent ainsi une nouvelle plasticité de la distribution des rôles entre acteurs privés et Etat dans la fonction de médiation des intérêts des consommateurs, 1'Etat se trouvant désormais concurrencé par les professionnels revendiquant eux-mêmes une nouvelle capacité à parler et agir « au nom du consommateur $»_{20}$.

\section{RÉFÉRENCES}

COCHOY (F.), 2002a. « Une petite histoire du client, ou la progressive normalisation du marché et de l'organisation », Sciences de la société, 44 (3), pp. 357-380.

COCHOY (F.), 2002b. Une sociologie du packaging ou l'âne de Buridan face au marché, Paris, PUF.

COCHOY (F.), GAREL (J.-P.), DE TERSSAC (G.), 1998. « Comment

l'écrit travaille l'organisation : le cas des normes ISO 9000 »,

Revue française de sociologie, 39 (4), pp. 673-699.

FRAENKEL (B.), 1995. « La traçabilité, une fonction caractéristique des écrits de travail », Connexions 65, pp. 60-75.

FRIEDBERG (E.), 1996. « Prescription et ordre moral ». In : DE TERSSAC (G.), FRIEDBERG (E.) (Ed), Coopération et conception, Octarès.

GRANJOU (C.), 2004. «La traçabilité, un dispositif de confiance dans les activités techniques. L'exemple de la filière viande bovine », Cahiers Internationaux de sociologie, 115, pp. 327-342. GRANJOU (C.), VALCESCHINI (E.), 2004. « Certifier en situation d'incertitude : le cas des OGM », Natures, Sciences et Sociétés, 4, pp. $404-412$.

GOODY (J.), 1986. La Logique de l'écriture. Aux origines des sociétés humaines, Paris, Armand Colin.

HOMMEL (T.), 2001. Environnement et stratégies des firmes industrielles. Le modèle de la gestion anticipative de la contestabilité appliquée à la production d'OGM agricoles et à l'industrie du traitement de surface en France et en Allemagne, Thèse du laboratoire d'économétrie de l'Ecole Polytechnique. KARPIK (L.), 1996. « Dispositifs de confiance et engagements crédibles », Sociologie du travail, 4, pp. 527-550.

ROT (G.), 1998. « Autocontrôle, traçabilité, responsabilité », Sociologie du travail, 1, pp. 5-20.

SAUVEE (L.), VALCESCHINI (E.), 2003. « Agro-alimentaire : la qualité au coeur des relations entre agriculteurs, industriels et distributeurs », In : Demeter 2004, Paris, Armand Colin.

TORNY (D.), 1998. «La traçabilité comme technique de gouvernement des hommes et des choses », Politix 44, pp. 51-75. VALCESHINI (E.), AVELANGE (I.), 2001. « Analyse économique et réglementaire de l'organisation d'une filière sans OGM ». Rapport du programme « Pertinence économique et faisabilité d'une filière sans utilisation d'OGM », INRA, Paris. 